\title{
SNS LLRF CONTROL SYSTEM MODEL DESIGN
}

\author{
Sung-il Kwon, Yi-Ming Wang, Amy Regan, LANL, Los Alamos, NM
}

Abstract- This paper addresses part of the design of the LLRF control system for the Spallation Neutron Source. Based on the Matlab/Simulink model of the Klystron and Cavity, considered as a two input two output (TITO) system, we design a PID controller which achieves the tracking of the set point reference. The PID controller design method modifies a relay-feedback-based PID auto-tuner for a single input single output (SISO) system. The original modeling was developed for the Low Energy Demonstration Accelerator and has been modified for the SNS. The advantage of this method is that the only system information required for tuning the PID controller gains is the oscillation gain (critical gain) and the oscillation frequency (critical frequency) from the relay-feedback control of the open loop system. From the oscillation gain (critical gain), we obtain the proportional gain, and from the oscillation frequency (critical frequency), we obtain the integration time and the derivative time by applying some algebraic rules.

\section{INTRODUCTION}

The low level RF(LLRF) control system for Spallation Neutron Source(SNS) is being designed at Los Alamos National Laboratory. The primary function of LLRF control system for SNS is to control RF fields in the accelerating cavity and maintain field stability within $\pm 1 \%$ peak to peak amplitude error and $1^{\circ}$ peak to peak phase error.

This paper addresses the problem of the LLRF control system for SNS. As an extension of the case of LEDA, we propose a PID controller. PID controller is the most popular controller for interacting multi-input multi-output systems in industries[1]. The main reason for this is its relatively simple structure which is easy to implement. In the case of actuator or sensor failure, it is relatively easy to stabilize manually. Despite the wide popularity of PID control, the number of applicable tuning methods is extremely limited. In this paper, we use auto-tuning of PID controller, which is a full extension of single-relay auto-tuner to the two input two output system[4]. The system assumptions are that first, the system is open-loop stable, second, the system has the low-pass characteristics. The klystron-cavity system meets this require- ments.

\section{KLYSTRON AND CAVITY MODEL}

The klystron is the most commonly used linear accelerator RF power source. The klystron used in SNS has two inputs, LLRF_I and LLRF_Q and two output HPRF_I and HPRF_Q. Let $u_{k}=\left[\begin{array}{ll}L L F_{-} I & L L R F \_Q\end{array}\right]^{T}$ and let $y_{k}=\left[\begin{array}{lll}H P R F_{-} I & H P R F_{-} Q\end{array}\right]^{T}$. The klystron in SNS is modelled as

$$
\begin{aligned}
& \dot{x}_{k}=A_{k} x_{k}+B_{k} u_{k} \\
& y_{k}=h\left(x_{k}, R(t)\right)
\end{aligned}
$$

where $A_{k} \in R^{2 \times 2}, B_{k} \in R^{2 \times 2}, x_{k} \in R^{2}$.

$h\left(x_{k}, R(t)\right) \in C^{2}$ is a continuous, nonlinear function vector of state $x_{k}$ and the high voltage power supply(HVPS) ripple $R(t)$. The details of the klystron model is given in [6].

The RF cavity can be expressed in the state space form.

$$
\begin{aligned}
& \dot{x}_{c}=A_{c} x_{c}+B_{c 1} u_{c 1}+B_{c 2} u_{c 2} \\
& y_{c}=C_{c} x_{c}
\end{aligned}
$$

where $u_{c 1}=\left[\begin{array}{lll}H P R F \_I & H P R F \_ & H_{-}\end{array}\right]^{T}, u_{c 2}=\left[B E A M \_I\right.$ $\left.B E A M_{-} Q\right]^{T}, y_{c}=\left[C A V \_F L D \_I C A V \_F L D \_Q\right]^{T}$, $A_{c} \in R^{2 \times 2}, B_{c 1} \in R^{2 \times 2}, B_{c 2} \in R^{2 \times 2}, C_{c} \in R^{2 \times 2}$, and $x_{c} \in R^{2}$. From the perspective of cavity, beam can be treated as an external disturbance for control purpose. Also, FLD_I and FLD_Q of sensor system are given by

$$
\left[\begin{array}{cr}
F L D \_I \\
F L D \_Q
\end{array}\right]=F A \cdot\left[\begin{array}{rr}
\cos (G D) & -\sin (G D) \\
\sin (G D) & \cos (G D)
\end{array}\right] y_{c}
$$

where $F A$ is an attenuation parameter and $G D$ is the phase offset of the open loop system. Series connection of the klystron and the cavity yields stable two input two output(TITO) nonlinear system.

\section{LLRF CONTROL STSTEM}

Consider a TITO system whose transfer function matrix $P(s)$ is given by

$$
P(s)=\left[\begin{array}{ll}
P_{11}(s) & P_{12}(s) \\
P_{21}(s) & P_{22}(s)
\end{array}\right] .
$$


The control matrix $C(s)$ is given by

$$
C(s)=\left[\begin{array}{cc}
C_{1}(s) & 0 \\
0 & C_{2}(s)
\end{array}\right]
$$

where $C_{i}, i=1,2$ are

$$
C_{i}(s)=K_{p}^{i}\left(1+\frac{1}{s T_{I i}}+\frac{s N_{i}}{s+\frac{N_{i}}{T_{D i}}}\right)
$$

and PID controller parameters $K_{P}^{i}, T_{I}^{i}, T_{D}^{i}, N_{i}, i=$ 1,2 are tuned by applying relay experiments.

Figure 1 shows the auto-tuning system for LLRF control system. STEAD1 and STEAD2 and two constant inputs are used for obtaining the steady state gain $P(0)$. RELAY1 and RELAY2 are used for obtaining the critical gains and the critical frequencies.

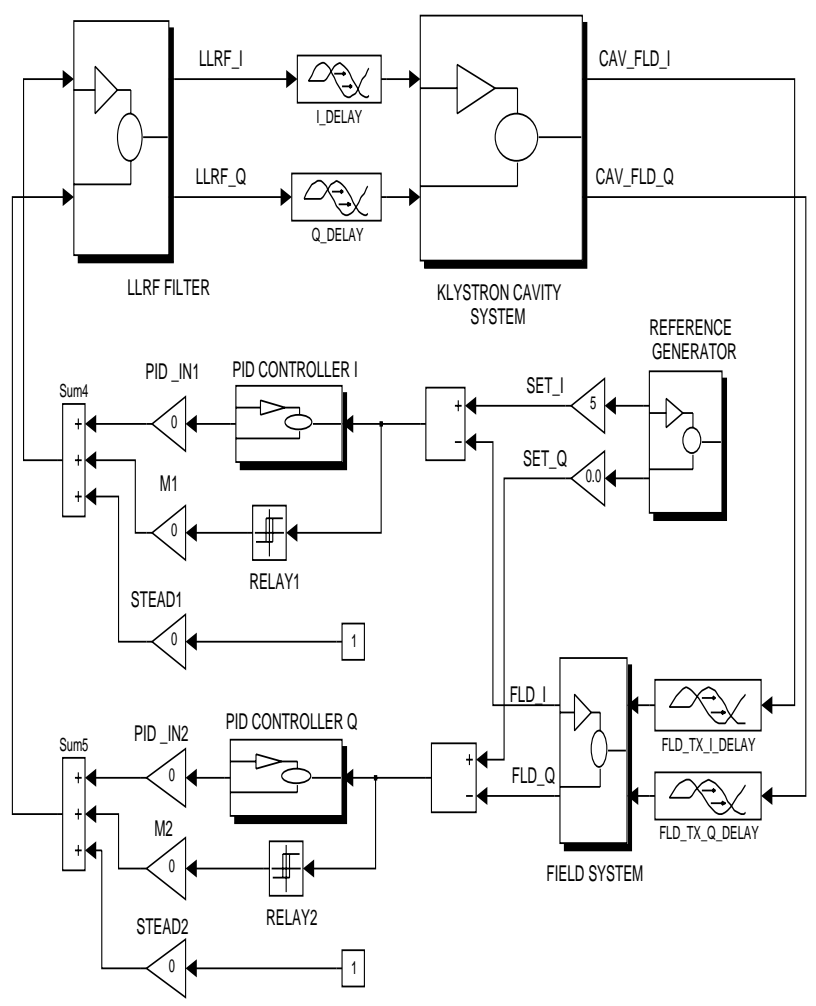

Figure 1. TITO LLRF decentralized relay system

In order to obtain the steady state gain $P(0)$, we disconnect unspecified PID controllers, two relays, RELAY1 and RELAY2. First, set the amplifier gain STEAD1 and reset amplifier gain STEAD2 and measure data FLD_I and FLD_Q. Second, set the amplifier gain STEAD2 and reset amplifier gain STEAD1 and measure data FLD_I and FLD_Q. From output data, we obtain the steady state gain $P(0)$ given by

$$
P(0)=\left[\begin{array}{ll}
\overline{F L D \_I}^{1} & \overline{F L D \_I}^{2} \\
\hline F L D \_Q & \overline{F L D \_Q}^{2}
\end{array}\right]
$$

where the superscripts indicate the simulation number and overline represents the steady state values of the outputs.
The next stage of simulations is obtaining the critical points of the system. In order to get the critical points, first, reset the amplifier gains STEAD1 and STEAD2 and connect RELAY1 and RELAY2. When the magnitudes of the relays are varying, the identified critical points move along the stability limits.

When the general describing function assumptions [3] are met, a good approximation of a critical point is given by

$$
\begin{aligned}
K_{i c r} & =\frac{4 M_{i}}{\pi a_{i}} \\
w_{i c r} & =\frac{2 \pi}{T_{i c r}}, \quad i=1,2 .
\end{aligned}
$$

where $M_{i}$ is the amplitude of the ideal relay and $a_{i}$ is the limit cycle amplitude. The critical frequency $w_{i c r}$ is calculated from the limit cycle time period which is determined by measuring the time between zero-crossings and the amplitude of oscillation is determined by measuring peak-to-peak value.

Then the desired critical point weighting $C_{d}$ which is defined by $\left.C_{d}=K_{2 c r}\left|P_{22}(0)\right| / K_{1 c r} \mid P_{11}(0)\right)$ between direct channels is given by

$$
\frac{M_{1}}{M_{2}}=\frac{1}{C_{d}} \frac{a_{1}}{a_{2}}\left|\frac{P_{22}(0)}{P_{11}(0)}\right| .
$$

The final stage is to tune the PID controller gains based on the critical points. A simple way is to choose a proportional gain with a rule

$$
K_{P}^{i}=\frac{K_{i c r}}{A_{m}^{i}}, \quad i=1,2
$$

where $A_{m}^{i}, i=1,2$ are the desired amplitude margins. In order to determine the integration time and the derivative time, we introduce a design parameter $\alpha_{i}$, $i=1,2$. Based on $\alpha_{i}$, we set $T_{I}^{i}, T_{D}^{i}, i=1,2$ as

$$
\begin{aligned}
& T_{I}^{i}=\alpha_{i} \frac{\pi}{w_{i c r}}, \\
& T_{D}^{i}=\frac{1}{w_{i c r}^{2} T_{I}^{i}}, \quad i=1,2 .
\end{aligned}
$$

Varying $\alpha_{i}, i=1,2$ within bounded sets, we can obtain $T_{I}^{i}$, and $T_{D}^{i}, i=1,2$.

\section{$4 \quad H_{\infty}$ CONTROL}

PID controllers yields good performance and robustness when the system perturbation is small. However, real world is different. It is apparent that the real klystron-cavity system has unmodelled dynamics, disturbances, sensor noises, electrical circuitry noises, which must be included in the model. For disturbances, we consider beam noise in beam system and high voltage power supply(HVPS) ripple in the klystron. Simulations[2] show that the effect of HVPS 
ripple is much more significant. For modelling uncertainties, we have to consider the ripple in frequency response and beam loading effect of the multi-pole klystron, and also we have to consider other modes in multi-cavities. These disturbances and model uncertainties affect the performance and stability of LLRF control system. When the uncertainties and the disturbances are small, then simple PID controllers with good stability margin can guarantee stability and performance of the LLRF control system. However, the real situation is not so positive. Simulation shows that $3 \%$ HVPS ripple degrades the LLRF control system performance significantly(phase error is serious). A remedy for this is an approach of $H_{\infty}$ controller. Modelling uncertainties, disturbances, noises, and desired performance specifications are characterized by weighting filters. Weighting filters are augmented to the nominal klystron-cavity system, which yields a generalized system. Then, we design a $H_{\infty}$ controller for the generalized system and is implemented for the real klystron-cavity system. We know that klystron is a nonlinear system and so the klystron-cavity system is nonlinear. This is mainly due to power saturation curve of the klystron. In order to capture the nonlinearity, a set of $H_{\infty}$ controllers are designed for each operating point and the scheduled controller is applied to the nonlinear klystron-cavity system, This is so called gain scheduling. When the operation range is small, then we can design one (robust) $H_{\infty}$ controller and apply the controller to the nonlinear klystron-cavity system. We design a $H_{\infty}$ controller having the property of gain scheduling, construct LLRF control system, and compare its performance with PID controller designed in previous sections. We assume that beam noise, sensor noise, and model uncertainty are ignorable and investigate HVPS ripple rejection(reduction). Figure 2 shows the LLRF control system with $H_{\infty}$ gain scheduling controller[2] and Figure 3 shows simulation results of open loop system, LLRF control system with PID controller, and with $H_{\infty}$ gain scheduling controller.

\section{REFERENCES}

[1] K. J. Astrom and T. Hagglund, PID controllers:theory, design, and tuning, 2nd edn. Instrument Society of America, 1995, Research Triangle Park, NC.

[2] Sung-Il Kwon, "Velocity Algorithm based on $H_{\infty}$ control for SN," preprint, LANSCE-5, RF Technology Group, LANL, 1999.

[3] I. J. Nagrath and M. Gopal, Control systems engineering, 2nd edition, John Wiley and Sons, Inc., New York, 1982.

[4] Z. J. Palmor, Y. Halevi, and N. Krasney, "Automatic tuning of decentralized PID controllers for TITO processes," Automatica, vol. 31, pp. 1001-1010, 1995.

[5] J. G. Ziegler and N. B. Nichols, "Optimum settings for automatic controllers," Trans. ASME, Vol. 64, pp. 759-768, 1942.
[6] C. Ziomek and A. Regan, "Simplification of Matrixx Model: Preliminary LLRF System Design" Technical Note, AOT-5TN:001, RF Technology Group, AOT Division, LANL, 1996.

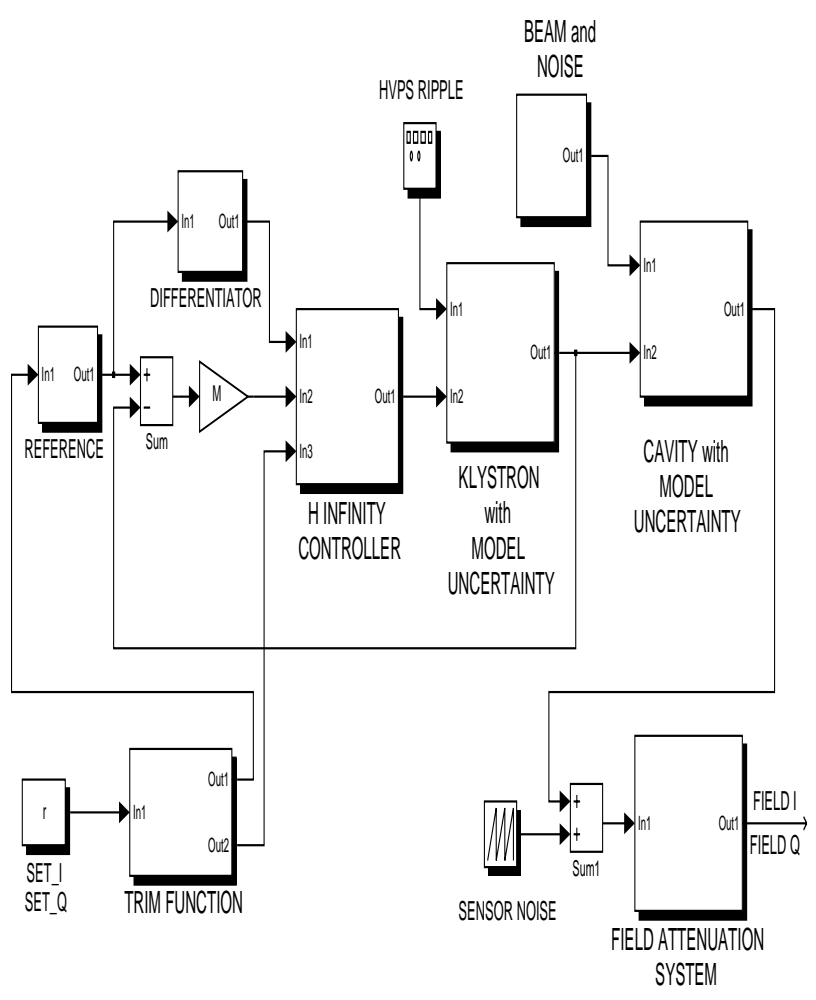

Figure 2. LLRF control system with gain scheduled $H_{\infty}$ Controller.
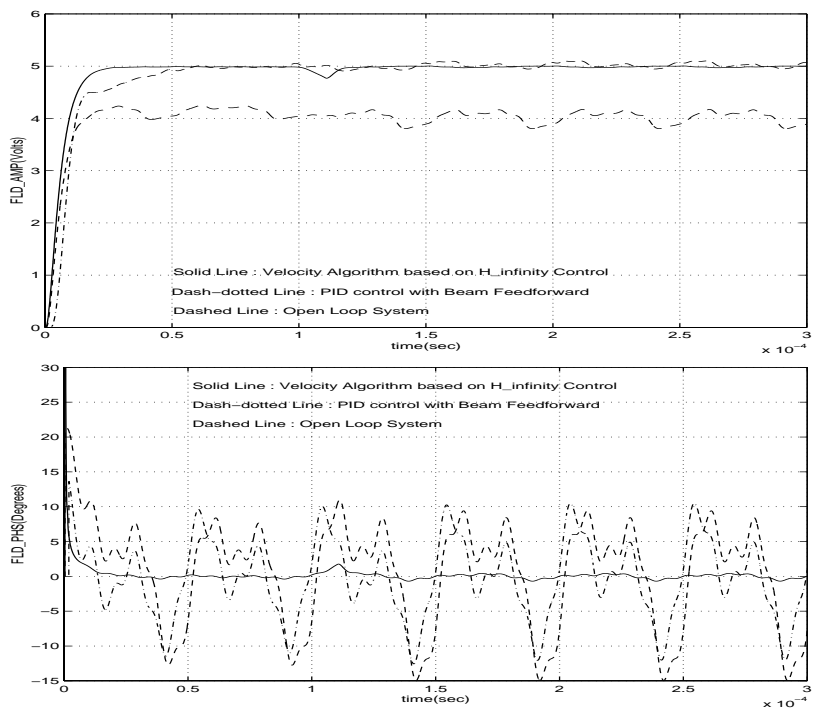

Figure 3. PID and $H_{\infty}$ Control System Responses with $3.0 \%$ HVPS ripple. Phase shift in klystron due to HVPS ripple is assumed to be $11.5^{\circ}$. 